\title{
A Rare Case of Triplet Pregnancy with Three Different Fates of each Conceptus
}

Paul S, ${ }^{1}$ Mandal S, ${ }^{1}$ Upadhyaya M, ${ }^{1}$ Pramanik SR, ${ }^{1}$

${ }^{1}$ Department of Gynaecology and Obstetrics, , IPGME\&R and SSKM HOSPITAL, Kolkata

Received: 23-May-2016; Accepted: 10-Dec-2016

\begin{abstract}
Rate and number of multifetal gestations have increased dramatically largely due to infertility therapy. A number of pregnancies are multifetal to start with followed by spontaneous reduction of one or more embryos. The final outcomes of such pregnancies vary in relation to zygosity, chorionicity and gestational age. In this case report we present a triplet pregnancy resulting from controlled ovarian hyperstimulation followed by intrauterine-insemination in a primarily infertile patient and how it ended up into a singleton pregnancy with no conscious attempts at selective reduction or selective termination. While fighting with problem of multiple pregnancies which comes along with the widespread infertility treatment nature has its own miraculous way to get rid of such. Little patience and watchful expectancy from the part of obstetrician can achieve a healthy live baby without inadvertent complication.
\end{abstract}

Keywords: Fetus papyraceus; multiple pregnancy; vanishing fetus

\section{INTRODUCTION}

Incidence of multiple pregnancies is rising with increasing use of ovarian hyperstimulation and assisted reproductive technology for the treatment of infertility. The actual rate of multiple pregnancies is significantly higher than that observed during labor due to intrauterine death of one or more fetuses in the course of pregnancy. There are three forms of this complication depending on the gestational period in which fetal death occurs, namely, vanishing fetus syndrome in first trimester; fetus papyraceus in second trimester and macerated fetus in third trimester. Prognosis of the surviving fetus depends on the chorionicity and the gestational age when the co-fetus dies.

\section{CASE}

A 33 years lady presented with primary infertility for six years with all baseline investigations including diagnostic laparoscopy within normal limits suggestive of unexplained infertility. After initial investigations controlled ovarian hyperstimulation(using sequencial clomiphene citrate and inj rFSH) with intrauterine insemination was planned. In the first cycle two dominant follicles were formed and ovulation was triggered using inj. Human chorionic gonadotrophin (10000 IU) followed by intrauterine insemination

\section{CORRESPONDENCE}

Dr. Sayanti Paul, Senior Resident

IPGME\&R and SSKM Hospital, Kolkata, West Bengal, India, Pin-711302

Email- ichchedana.sayanti@gmail.com

Phone- +919836799167 using husbands semen. Pregnancy was diagnosed and ultrasonography revealed three embryos with cardiac activities and of $7 \mathrm{wks}, 6 \mathrm{wks} 5$ days and $6 \mathrm{wks}$ 4 days gestational age respectively. Second USG was done after 6 weeks. It showed two living fetus of 13 wks 3 days and 13 wks 5 days gestational age with two separate placentas and one dead fetus of 7 wks5 days. Anomaly scan shows no gross anomaly. Rescan at 28 weeks revealed one live fetus of 27 weeks gestational age and another dead fetus of 23 weeks 4days with spalding sign. There was no trace of third fetus (vanishing fetus). Considering the early weeks of second fetal death and dichorionic nature of the pregnancy we decided conservative management with weekly monitoring of coagulation profile and fetal growth by USG. Mother was given single course of betamethasone injection. At 34 weeks of gestation the pregnancy was terminated by emergency caesarean section following preterm premature rupture of membrane and a healthy living boy baby was delivered ( $2 \mathrm{~kg} 100 \mathrm{gms})$. Another male fetus papyraceus was found stuck to the placenta (as shown in Figure 1). The placenta was dichorionicdiamniotic. The patient with baby was discharged on sixth postoperative day with uneventful postpartum period.

\section{COMMENTS}

Multiple pregnancy is a frequent complication associated with ovarian hyperstimulation (15-20 $\%$ of pregnancies twins and $5 \%$ triplets or more). ${ }^{1-}$

3 Though low-dose follicle stimulating hormone 
(FSH) regimens have succeeded in reducing the rate of multiple pregnancies to $2-6 \%{ }^{3}$ In the reported case the patient was treated with $\mathrm{CC}$ and inj. $\mathrm{rFSH}$ sequencially followed by IUI when two DF developed and it resulted in a dichorionic triplet pregnancy and finally ended in a singleton live birth.

Figure 1: Figure showing a fetus papyraceus covered in amniotic membrane stuck to placenta which was found following delivery of the live baby

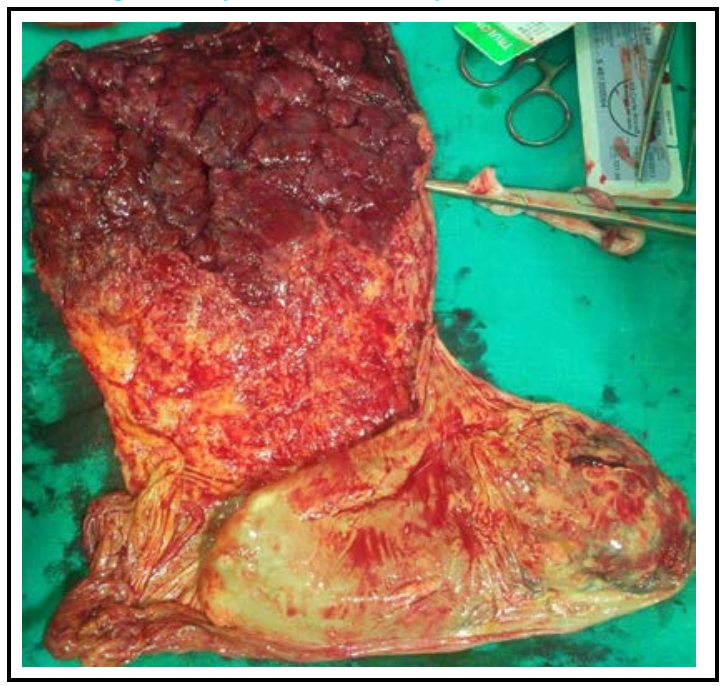

Stoeckel (1945) was the first to suggest that the conception rate of multiple gestations was greater than the birth rate. Four decades later, ultrasound enabled visualization and confirmation of this event, now characterized as the 'vanishing twin' phenomenon. The process of early pregnancy disappearance appears to involve resorption and/or formation of a blighted ovum. The true incidence of vanishing concepti is difficult to assess. Reported frequencies range from 3.7 to $100 \%$. The majority of pregnancies identified with twin sacs or embryos $(\sim 61 \%)$ continued as twin gestations whereas those with three sacs or embryos more frequently resulted in twin or singleton gestations. Disappearance of sacs or embryos has been described throughout the first trimester, occurring more frequently earlier in time. ${ }^{4-7}$ In the report of triplet sacs and embryos by Manzur et al. (1995), almost 90\% of resorptions occurred in the first 7 weeks of gestation. ${ }^{7}$ Spontaneous reduction has not been reported to occur beyond 13 weeks of gestation. ${ }^{4,7}$ In this case first sonogram revealed all three foetuses with good cardiac activity at approx. 7weeks of gestational age but repeat sonogram at 13 weeks showed death of the third fetus. As discussed already future sonograms failed to detect any trace of the vanished fetus. Following delivery there was no obvious placental signs on gross examination suggestive of vanishing conceptus.

After 8 weeks the death of one embryo in a multiple pregnancy, with resorption of amniotic fluid and mummification of the fetal parts, will cause a fetus papyraceus. Delivery of a fetus papyraceus indicates death must have occurred at least ten weeks previously. Unless sufficient time has elapsed for mummification, the dead fetus will be delivered as a macerated stillbirth rather than a fetus papyraceus. In the index case the second fetus died at around 23 weeks of gestational age whereas caesarean section was done at 34 weeks. So the scenario was ideal for development of fetus papyraceus as discussed above. The index case presented with preterm premature rupture of membrane with no other complication and caesarean section was done considering the background history of long primary infertility and the risks. Though coagulation profile was monitored at regular interval there was no sign of coagulopathy. The prognosis of the surviving fetus depends on the chorionicity. Often the surviving monochorionic twin of a fetus papyraceus is prone to neurologic complications, cerebral palsy, renal failure and intrauterine or neonatal deaths. In dichorionic pregnancy the prognosis is much better and most frequent complication being the prematurity. In the reported case there was no such complication due to the dichorionicity. Earlier reports suggested that fetus papyraceus occurs more frequently in monochorionic than in dichorionic twin pregnancies. However, according to Benson et al in $\mathrm{DC}(12 \%)$ and $\mathrm{MC}$ (11\%) pregnancies frequency is similar. ${ }^{6}$

Management of early intrauterine fetal loss in a multiple pregnancy with one surviving fetus should be conservative with an aim to delay delivery atleast till the living fetus can survive ex-utero and at the same time not to compromise maternal health or increase the morbidity of the living fetus. So it needs specialist obstetric care in the tertiary referral centre. It is also necessary to document the intrauterine fetal death allowing legal protection against the accusation of malpractice and having caused neurologic damage to the child during birth. 


\section{REFERENCES}

1. Crosignani PG, Somigliana E. Effect of GnRH antagonists in FSH mildly stimulated intrauterine insemination cycles: a multicentre randomized trial. Hum Reprod. 2007;22(2):5005.

2. Khalil MR, Rasmussen PE, Erb K, Laursen SB, Rex S, Westergaard LG. Homologous intrauterine insemination. An evaluation of prognostic factors based on a review of 2473 cycles. Acta Obstet Gynecol Scand. 2001;80(1):74-81.

3. Tur R, Barri PN, Coroleu B, Buxaderas R, Martínez F, Balasch J. Risk factors for high-order multiple implantation after ovarian stimulation with gonadotrophins: evidence from a large series of 1878 consecutive pregnancies in a single centre. Hum Reprod Oxf Engl. 2001;16(10):2124-9.
4. Blumenfeld Z, Dirnfeld M, Abramovici H, Amit A, Bronshtein M, Brandes JM. Spontaneous fetal reduction in multiple gestations assessed by transvaginal ultrasound. $\mathrm{Br} \mathrm{J}$ Obstet Gynaecol. 1992;99(4):333-7.

5. Sampson A, de Crespigny LC. Vanishing twins: the frequency of spontaneous fetal reduction of a twin pregnancy. Ultrasound Obstet Gynecol Off J Int Soc Ultrasound Obstet Gynecol. 1992;2(2):107-9.

6. Benson CB, Doubilet PM, Laks MP. Outcome of twin gestations following sonographic demonstration of two heart beats in the first trimester. Ultrasound Obstet Gynecol Off J Int Soc Ultrasound Obstet Gynecol. 1993;3(5):343-5.

7. Manzur A, Goldsman MP, Stone SC, Frederick JL, Balmaceda JP, Asch RH. Outcome of triplet pregnancies after assisted reproductive techniques: how frequent are the vanishing embryos? Fertil Steril. 1995;63(2):252-7. 